\title{
POSITRONIUM FRACTION AND POSITRON LIFETIME AT Ni(110) AND Ge(110) SURFACES
}

\author{
S.B. Shrivastava and V.K. Gupta \\ School of Studies in Physics, Vikram University, Ujjain 456 010, India \\ (Received September 4, 1989; in final form September 4, 1990)
}

The fraction of positronium formation and the positron lifetime at $\mathrm{Ni}(110)$ and $\mathrm{Ge}(110)$ surfaces, when low-energy positrons incident on them, have been calculated using the rate equations approach and positron trapping in image potential well. The calculated results are compared with the available experimental results. The positronium fraction is overestimated at high temperatures in case of $\mathrm{Ni}(110)$.

PACS numbers: 68.35.-p, 25.30.Hm

\section{Introduction}

In recent years the properties of solid surfaces have been investigated very widely with the help of monoenergetic positron beams $[1,2]$. Such studies provide information about the surface states and help to understand the processes involved when a beam of slow positron strikes a solid surface. In a series of experiments Mills, Lynn and their collaborators [3-5] have studied the dynamics of positrons and the formation of positronium at surfaces in a number of metals using a beam of slow positrons incident on metal surfaces. A striking result of these experiments is that a significant percentage of all the positrons seems to be annihilated as positronium. In these experiments a monoenergetic beam strikes a well characterized single crystal surface. The majority of positrons penetrate the solid and simultaneously lose their energy rapidly with a characteristic stopping distance. A large fraction of these thermalized particles eventually diffuse back to the entrance surface. The positron reaching the surface may capture an electron at the surface and thus emerges as positronium rather than a free positron. Other possibilities are the trapping of the positrons into surface states. The above cited authors have measured the fraction of positronium formation as a function of temperature and the incident energy. 
Different mechanisms have been proposed to explain these results by various authors [ 6,7$]$. For example Chu et al. [7] have discussed the problem of positronium emission from metal surface and derived an equation on the basis of thermodynamics, similar to that of Richardson Dushman for positronium emission. Kreuzer et al. [6] have calculated the fraction of positrons forming positronium at a metal surface by solving rate equations. Recently Nieminen and Puska [8] have developed a computational scheme for obtaining the positron states and their annihilation characteristics. They have also calculated the lifetime of positrons for certain simple metal surfaces. Bhadoria and Shrivastava have discussed the mechanism of positron annihilation following a rate equation approach $[9,14]$. The model was used to obtain the positron lifetimes at different surfaces of $\mathrm{Al}, \mathrm{Au}$ and Ag. Earlier we had extended the above model to calculate the fraction of positronium formation at metal surface as a function of temperature [10]. In the following we present the results of the calculation of positronium fraction and the lifetime of positrons at the surfaces of $\mathrm{Ni}(110)$ and $\mathrm{Ge}(110)$ employing the above model of Shrivastava et al. [10]. The purpose is to apply the model to non-simple metals to understand the positron annihilation at the surfaces of these metals.

As pointed out in Ref. [10] the calculation approach is based on the following main processes supposed to occur when a low-energy positron is incident on a metal surface.

(i) The positrons are annihilated via positronium formation at a metal surface, which is different from the characteristic annihilation in bulk, where positronium formation is inhibited due to screening of conduction electrons.

(ii) As the positron leaves the surface it sees an effective potential well near a surface due to image forces at large distances and due to correlation energy with the low-density electron gas at short distances outside the last atomic layer. The positron states in this potential well form a set of two-dimensional energy bands [1]. The positron may undergo elastic collisions and may be trapped in these surface states. Once in the well, the positron can either annihilate or escape as free positronium if sufficient energy is available due to thermal fluctuations.

(iii) The positrons can also be directly re-emitted from metal into the vacuum in those materials which have a negative positron work function or the re-emission in the vacuum occurs as a positronium atom. We assume that the incident energy of the positrons is sufficiently low $(100 \mathrm{eV})$ so that almost all positrons annihilate from surface states and that positron trapping at thermally generated monovacancies is not observed.

On the basis of the above processes, as a function of time, we can write a set of simple equations for their rate of change:

$$
\begin{gathered}
\frac{\mathrm{d}}{\mathrm{d} t} n_{s}(t)=-\left(\alpha_{e^{+}}+\alpha_{p s}+\alpha_{S T}\right) n_{s}(t), \\
\frac{\mathrm{d}}{\mathrm{d} t} n_{T}(t)=\alpha_{S T} n_{s}(t)-\left(\lambda_{c}+\lambda_{i}+\alpha_{T p}\right) n_{T}(t),
\end{gathered}
$$




$$
\begin{aligned}
\frac{\mathrm{d}}{\mathrm{d} t} n_{p s}(t) & =\alpha_{p s} n_{s}(t)-\dot{\lambda}_{p} n_{p s}(t), \\
\frac{\mathrm{d}}{\mathrm{d} t} n_{T p}(t) & =\alpha_{T_{p}} n_{T}(t)-\lambda_{p} n_{T p}(t) .
\end{aligned}
$$

Here $n_{s}(t)$ is the fraction of positrons at the surface at any time $t, n_{T}(t)$ is the fraction of positrons trapped in the surface states, $n_{p s}(t)$ is the fraction of positrons forming positronium in vacuum by escaping from surface and $n_{T_{p}}(t)$ is the fraction of positronium by escaping from the trapped region. The rates $\lambda_{c}, \lambda_{i}$ are annihilation rates in the surface states arising due to electron correlation just near the surface and due to image potential at large distances, respectively. $\lambda_{p}$ is the spin averaged annihilation rate of positronium atom.

In Eq.(1) the escape rate at the surface is [3]:

$$
\alpha=\alpha_{e+}+\alpha_{p s}+\alpha_{S T}
$$

where the emission rate as a free positron

$$
\alpha_{e+}=(1-P) \alpha_{0}=y_{0} \alpha_{0}
$$

and that as a free positronium

$$
\alpha_{p s}=P \alpha_{0}
$$

and $\alpha_{S T}$ is the capture rate into the surface state. In the above equations, $\alpha_{0}$ is the intrinsic penetration rate and $P$ is the conversion probability for the positrons leaving the surface to form positronium. In Eq. (2), $\alpha_{T_{p}}$ is the thermal desorption rate produced when positrons are thermally desorbed from surface states and form positronium while escaping from the surface. Mills and Murry [11] obtained an approximate formula for calculation of $P$

$$
P \approx 1-\exp \left(-\sqrt{E_{0} /\left|\psi_{+}\right|}\right),
$$

where $E_{0}$ is the band shift energy and $\psi_{+}$is the positron work function.

Equations (1-4) have been solved for the appropriate boundary conditions. We assume initially that no positrons are trapped in the image potential or form positronium.

The solutions so obtained are used to calculate the lifetime of positrons and the fraction of positronium formation at a metal surface (for details see [10]):

$$
\begin{gathered}
\tau_{S}(T)=\int_{0}^{\infty}\left[n_{s}(t)+n_{T}(t)+n_{p s}(t)+n_{T p}(t)\right] \mathrm{d} t \\
\tau_{S}(T)=\frac{\frac{P \alpha_{0}}{\alpha_{S T}}+\frac{\alpha_{T p}}{\left(\lambda_{c}+\lambda_{i}+\alpha_{T p}\right)}}{\left[1+\frac{\alpha_{0}}{\alpha_{S T}}\right] \lambda_{p}} .
\end{gathered}
$$

The fraction of positronium formation is given by:

$$
\begin{array}{r}
f_{P s}=\int_{0}^{\infty} \lambda_{p}\left[n_{p s}(i)+n_{T p}(t)\right] \mathrm{d} t \\
=\frac{\frac{P \alpha_{0}}{\alpha_{S T}}+\frac{\alpha_{T_{p}}}{\left(\lambda_{c}+\lambda_{i}+\alpha_{T p}\right)}}{1+\frac{\alpha_{0}}{\alpha_{S T}}} .
\end{array}
$$


The transition rate out of the surface trap or detrapping rate is given by [14]:

$$
\alpha_{T p}=\nu \exp \left[-E_{a} / k T\right]=A T \exp \left[-E_{a} / k T\right]
$$

where $\nu$ is a frequency factor of the bound positron in the surface states and is found to be proportional to temperature. $E_{a}$ is the activation energy and $A$ is the constant of proportionality.

In the present work the values of $\alpha_{S T}$ and $\alpha_{0}$ are selected with a view to be consistent with the experimental results. We found that in order to get the best results $\alpha_{S T}$ shuold be equal to $1 \times 10^{14} \mathrm{~s}^{-1}$ and $\alpha_{0}$ should be equal to $0.7 \times 10^{14} \mathrm{~s}^{-1}$ for $\mathrm{Ge}$ and $0.5 \times 10^{14} \mathrm{~s}^{-1}$ for $\mathrm{Ni}$.

\section{Results}

Employing the procedure as described above, the positron lifetime and the fraction of positronium formation at metallic surface of $\mathrm{Ge}(110)$ and $\mathrm{Ni}(110)$ have been calculated using Eqs. (10) and (11). The various parameters used in the calculations are listed in Table $I$ along with the source from which these have been taken. The calculated values are given in Table II. In the same table we have compared our results with the available experimental results.

\section{TABLE I}

The list of parameters used in calculations.

\begin{tabular}{c|c|c|c|c}
\hline \hline Parameter & $\mathrm{Ge}(110)$ & Ref. & $\mathrm{Ni}(110)$ & Ref. \\
\hline$A$ & $6.26 \times 10^{11}$ & 10 & $6.26 \times 10^{11}$ & 10 \\
$E_{a}(\mathrm{eV})$ & 0.83 & 1 & 0.75 & 1 \\
$P$ & 0.87 & $*$ & 0.87 & $*$ \\
$\alpha_{S T}\left(\mathrm{~s}^{-1}\right)$ & $1.0 \times 10^{14}$ & $*$ & $1 \times 10^{14}$ & $*$ \\
$\alpha_{0}\left(\mathrm{~s}^{-1}\right)$ & $0.7 \times 10^{14}$ & $*$ & $0.5 \times 10^{14}$ & $*$ \\
$\lambda_{c}\left(\mathrm{~s}^{-1}\right)$ & $1.5 \times 10^{9}$ & $*$ & $2.5 \times 10^{9}$ & $*$ \\
$\lambda_{i}\left(\mathrm{~s}^{-1}\right)$ & $4.47 \times 10^{8}$ & $*$ & $6.3 \times 10^{8}$ & $*$
\end{tabular}

* Present work

The calculated positronium fractions at metallic surfaces of $\mathrm{Ni}(110)$ and $\mathrm{Ge}(110)$ have been plotted as a function of temperature in Figs. 1 and 2 respectively. Figure 1 shows that the positronium fraction is constant up to $500 \mathrm{~K}$ and then it increases with temperature. On comparing these data with the experimental results of Koymen and Gidley [12] (Table II) we find that calculated results are in good agreement at low temperature. However they are overestimated at higher temperature. 


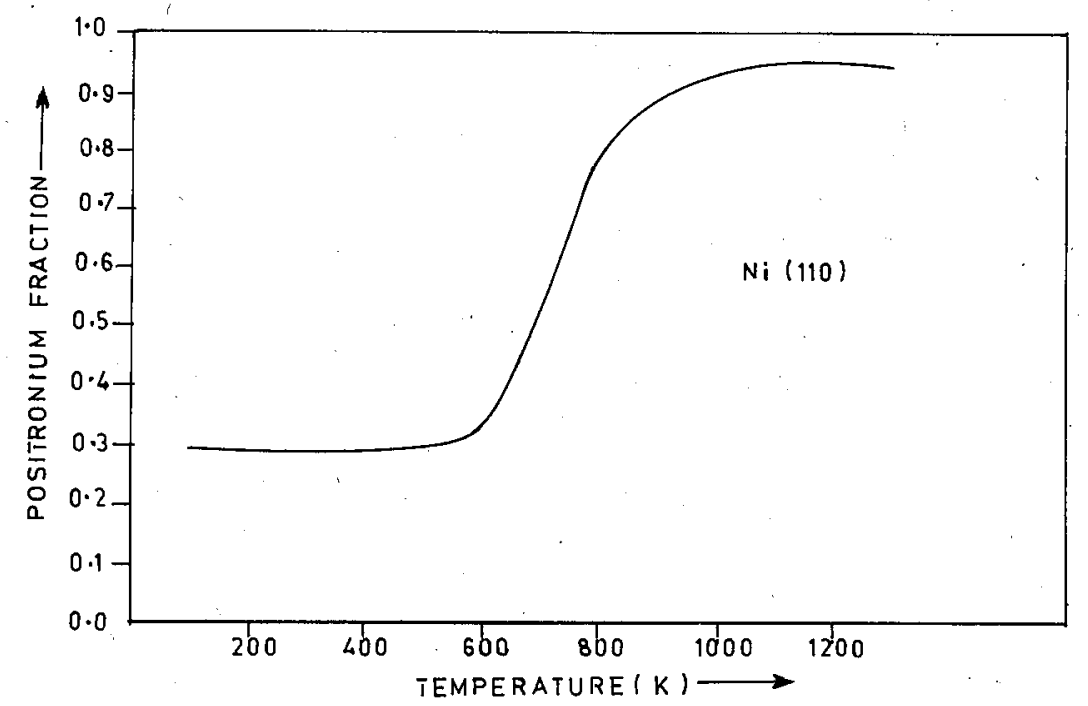

Fig. 1. Calculated positronium fraction as a function of temperature at $\mathrm{Ni}(110)$ surface.

TABLE II

\begin{tabular}{c|c|c}
\multicolumn{3}{c}{ Comparision of positronium fraction at Ni(110). } \\
\hline \hline $\begin{array}{c}\text { Temperature } \\
\text { (in K) }\end{array}$ & $\begin{array}{c}\text { Calculated } \\
\text { values }\end{array}$ & $\begin{array}{c}\text { Experimental values } \\
\text { Ref.[12] }\end{array}$ \\
\hline 300 & 0.290 & 0.270 \\
400 & 0.290 & 0.275 \\
500 & 0.292 & 0.295 \\
600 & 0.332 & 0.31 \\
700 & 0.547 & 0.33 \\
800 & 0.804 & 0.37 \\
900 & 0.908 & 0.44 \\
1000 & 0.939 & 0.485
\end{tabular}

In case of $\mathrm{Ge}(110)$ (Fig. 2), the positronium fraction $P s$ is constant at low temperatures up to $600 \mathrm{~K}$ and then it increases almost linearly with temperature up to $1100 \mathrm{~K}$ and after that it becomes nearly constant. These results have been compared with the available experimental results of Jorch and Lynn [13] in Table III. We have found that the calculated results agree well with the experimental ones. The calculated lifetime of positrons at metal surfaces of $\mathrm{Ni}(110)$ has been plotted as a function of temperature in Fig. 3. This could be compared well with the experimental lifetime of Naguchi et al. [15] in fine metal powders, which is $386 \mathrm{ps}$ at room temperature, showing that in fine powders surface effects are predominant. 


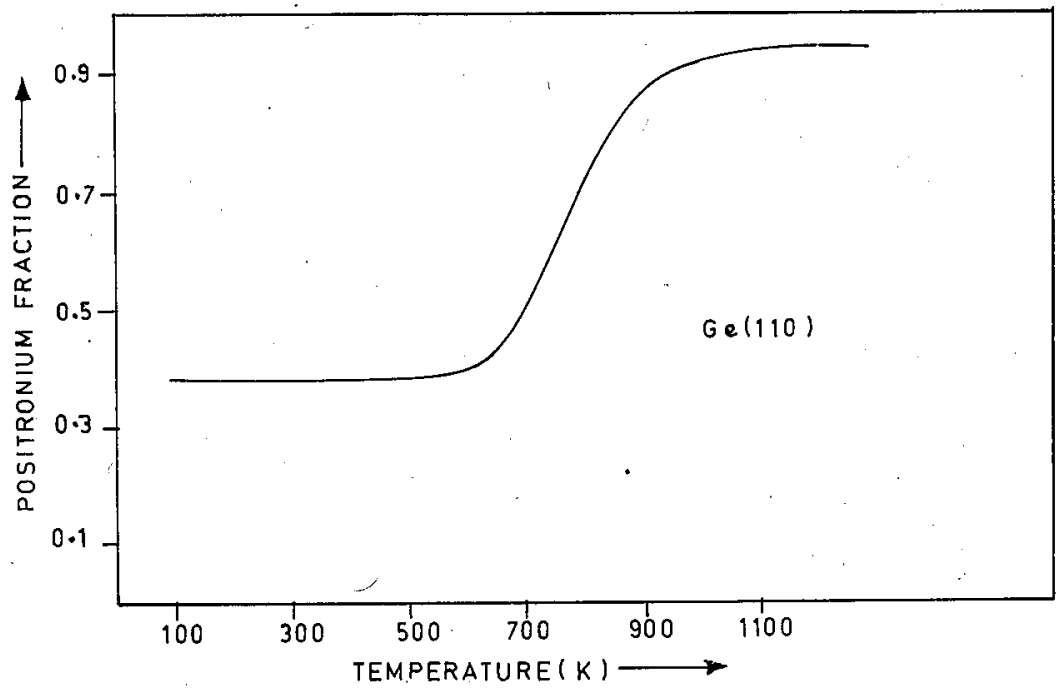

Fig. 2. Calculated positronium fraction as a function of temperature at Ge(110) surface.

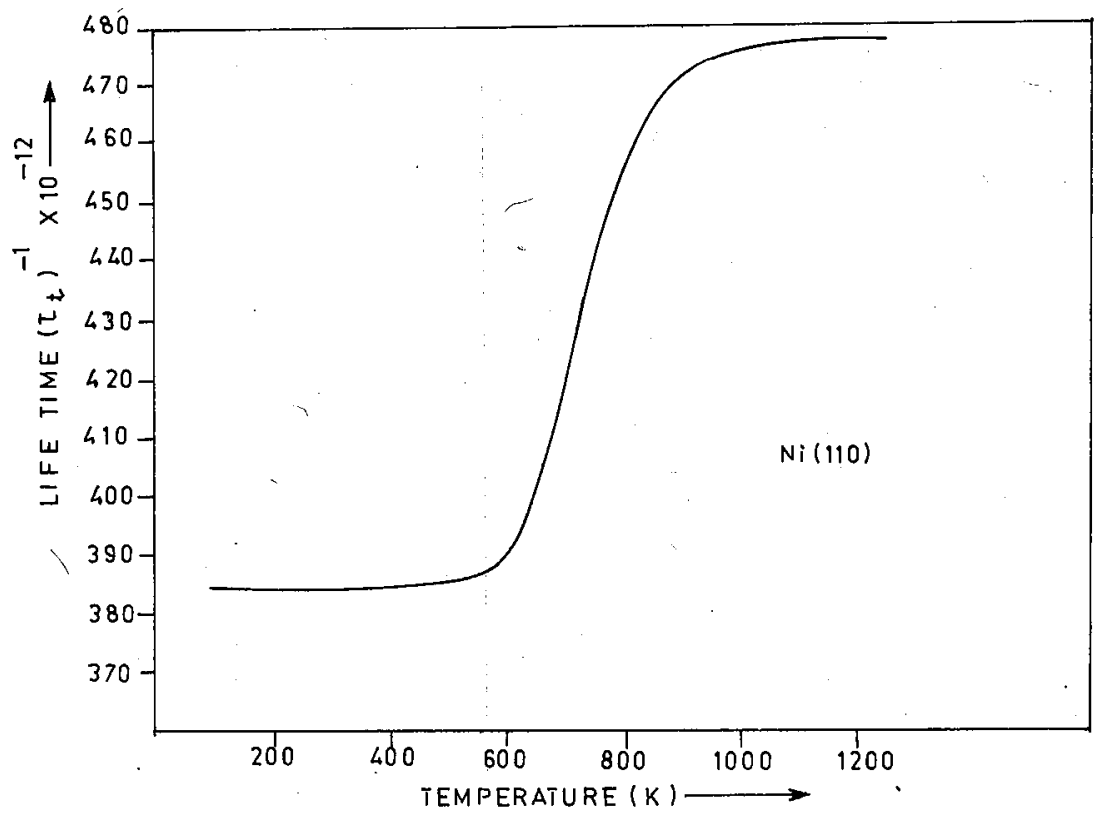

Fig. 3. Calculated positron lifetime vs. temperature at $\mathrm{Ni}(110)$ surface. 
TABLE III

Comparison of calculated values of positronium fraction at $\mathrm{Ge}(110)$ with the available experimental values.

\begin{tabular}{c|c|c}
\hline \hline $\begin{array}{c}\text { Temperature } \\
\text { (in K) }\end{array}$ & $\begin{array}{c}\text { Calculated } \\
\text { values }\end{array}$ & Experimental values \\
\hline 341 & 0.38 & 0.36 \\
985 & 0.90 & 0.86
\end{tabular}

In case of $\mathrm{Ge}(110)$ the calculated values of lifetimes do not show much variation with temperature and are around $476 \mathrm{ps}$ at room temperature. Thus the above model may not lead to very satisfactory results in case of semiconductors, where other effects like positron diffusion etc. are important. More theoretical and experimental effort is needed to verify the model.

\section{References}

[1] A.P. Mills Jr., in Positron Solid State Physics, eds. W. Brandt, A. Dupasquier, North Holland, Amsterdam 1983, p. 43.

[2] A.P. Mills Jr., in Positron Annihilation, eds. P.G. Coleman, S.C. Sharma, L.M. Diana, North Holland, Amsterdam 1982, p. 121.

[3] K.G. Lynn, D.O. Welch, Phys. Rev. B 22, 99 (1980).

[4] K.G. Lynn, H.L. Lutz, Phys. Rev. B 22, 4143 (1980).

[5] K.G. Lynn, J. Phys. C 12, L 435 (1979).

[6] H.J. Kreuzer, D.N. Lowy, Z.W. Gortel, Solid State Commun. 35, 781 (1980).

[7] S. Chu, A.P. Mills Jr., C.A. Murray, Phys. Rev. B 23, 2060 (1981).

[8] R.M. Nieminen, M.J. Puska, Phys. Rev. Lett. 50, 281 (1980).

[9] K.S. Bhadoria, S.B. Shrivastava, Solid State Commun. 53, 391 (1985).

[10] S.B. Shrivastava, V.K. Gupta, K.S. Bhadoria, Phys. Status Solidi B 147, 553 (1988).

[11] A.P. Mills Jr., C.A. Murray, Bull. Am. Phys. Soc. 75, 392 (1980).

[12] A.K. Koymen, D.W. Gidley, Phys. Rev. B 35, 1034 (1987).

[13] H.H. Jorch, K.G. Lynn, Phys. Rev. B 30, 93 (1984).

[14] A.P. Mills Jr., Solid State Commun. 31, 623 (1979).

[15] S. Noguchi, Y. Miyata, in Proc. 5th Int. Conf. Positron Annihilation, eds. R.R. Hasiguti, K. Fujiwara, (Sendai, Japan), 1979, p. 11B-III-2. 